\title{
Concept, Analysis and Design of an Adaptive Rehabilitation Technology through Cognitive Effort Analysis
}

\author{
Gahangir Hossain* \\ Texas A\&M University Kingsville, USA
}

Submission: February 16, 2018; Published: March 12, 2018

*Corresponding author: Gahangir Hossain, Texas A\&M University-Kingsville, Texas, USA, Email: Gahangir.Hossain@tamuk.edu

\begin{abstract}
Individuals with disability (e.g. visual impaired) are unique in their own ways. Existing solutions are often inadequate in effectively assisting them in their need. Assistive thinking is a paradigm shift in the design and implementation of intelligent technology solutions for individuals with visual impairment or blindness, which can be applied in other disabilities. It calls for an in-depth analysis of a user's need as well as preference, estimate the cognitive ability-vs-demand gap from user's mental effort, and integrate user requirements using design and systems thinking ideas into the assistive technology (AT) systems. In addition, it advocates an effective use of existing resources in managing disability with a sustainability plan to maximize the utility of technology solutions with adaptive user interaction. This is expected to transform the design, implementation, and use of assistive systems that not only meet users' needs, but also address their situational and social needs. This paper presents the culminating experiences of designing assistive systems and a pilot study that was performed using a set of usability questions and observed cognitive capabilities of representative users, the persons who are blind or visual impaired. Assistive thinking considers a user's activity pattern based on the interface design pattern, analyzes cognitive load during technology interaction, communicate directly to technology and generate feedback to the user. It also addresses the user's unintended consequences that may cause failure and facilitates subsequent modeling to improve technology performance resulting both accessible and useable interfaces for people with disabilities.
\end{abstract}

Keywords: Intelligent Systems; Adaptive systems; assistive systems; systems thinking; User interaction; User activity modeling; assistive user interface; design thinking; Cognitive efforts; Cognitive ability-demand gap; cognitive load, Assistive Technology; mobile assistive technology; assistive thinking

Abbreviations: AT: Assistive Technology; SD: Systems Dynamics; UCD: User-Centered Design; SOS: System of Systems; HAAT: Human Activity Assistive Technology; SE: Systems Engineering; LCS: Lip Control System; CLDs: Causal Loop Diagrams; USID: User Sensitive Inclusive Design; UCD: Centered Design; CLD: Causal Loop Diagram; CLST: Cognitive Load Systems Thinking; CLDT: Cognitive Load Assessed through Design Thinking

\section{Introduction}

Recent years have witnessed an explosion of interest in mobile application development. As of now a total of 170,000 apps were published on Google's Android Market by 2015'. The question persists whether the growth rate of android applications will enhance the quality of life and social interaction of people with disability, especially individuals with visual impairment. Despite a huge number of mobile applications (apps) are in the market and the number increasing day by day, to have a user-centered intelligent application, it requires an in depth analysis of users' mental state in addition to the systems and design thinking. The key idea is to keep the design very simple, as the user might like, so that the user can interact with the system effectively with minimal cognitive effort. According to US public law, an assistive technology (AT) system is defined as the element or product system, whether

${ }^{1}$ Android Apps: https://play.google.com/store

2Definition of Disabilities, Disabled World - www.disabled-world.com, 2014. acquired commercially off the shelf, modified or customized, that is used to increase or improve functional capabilities of individuals with disabilities ${ }^{2}$. A novel purpose of designing AT is to narrow down the cognitive gap between the level a person with disability works, but at the level he should perform. While designing an AT, it is important to nurture it's users' higher-level thinking skills that commensurate with their technology skills and/or cognitive level, which often measure at a significantly higher level than their functional level and performance. As an example, a braille display used by a Blind people can be improved through integration with cognitive gap enabled smart phone applications. Hence, the purpose is to develop AT application and services based on person's cognitive ability-demand gaps, that were not considered in original design. Designing a user-centered AT is therefore a 
challenging problem, which requires a higher level of thinking instead of the traditional methods of design and implementation. This recalls the Albert Einstein's famous quote, "Problems cannot be solved by the same level of thinking that created them". In this context (e.g., Mobile assistive application design), a rethinking in the design and development of applications (e.g. Android apps (Rosener 2012) and their dissemination with the system is a necessity and an integral part of developing assistive solutions and disability management.

Two prominent thinking approaches are systems thinking and design thinking. Systems thinking allows examining how engineers create new systems, seeing the large picture, and recognizing whether the structure improves performance [1]. Design thinking estimates how alternative sets of user behavior patterns would serve set of goals in such a way that make the user think in meeting his requirements [2,3]. To summarize, systems thinking helps us understand the problems more deeply [4] before jumping to a quick solution, while the design thinking helps us to create unique and often non-obvious solutions useful to target users [5]. This work proposes a paradigm shift, called assistive thinking [3], to address issues with current practices in AT applications and to have a smart, robust and AT solutions. It is a holistic approach for designing assistive solutions that include (but are not limited to):

a) Understanding users cognitive ability-demand gap in terms of cognitive effort;

b) Requirement analysis to understand user's needs and preferences from pattern of accessibility;

c) Integrating systems and design thinking, to develop technology solutions that accounts for users cognitive gap as well as preferences;

d) Follows iterative and participatory design to bridge the gap of the user in technology adaptation; and

e) Provides sustainable plan for the effective use of existing resources in managing disability to maximize the utility of technology solutions.

It might be useful to consider a graphical model, thinking of the process as having different paths depending on the results of iterative analysis [6] So the types of evaluations of the design might change depending on user feedback.

The key idea is to integrate two popular design principles:

I. Systems thinking [7] and

II. Design thinking [8] with the user's mental model (Kiselev \& Loutfi 2004) (e.g. Iceberg model $[9,10]$ ) that will bridge ability-demand gap of a user while using assistive solutions.

Generally, systems thinking if a futuristic thinking process that aims to construct the system such a way to improve the system in future. Alternatively, the design thinking considers designers' approaches in the creative use of the technology tool. In this pilot study, different types of communication gaps that arise in human-technology interaction are analyzed, in terms of cognitive ability-demand gap [11,12]. Only the cognitive abilitydemand gap was considered, so the systems dynamics (SD) model was used to simulate the scenario. Two sets of subjective (NASATLX) [13] rating data are used to understand users' cognitive load, while interacting with the system (in our case R-MAP $[14,15]$ ) and Nielsen's usability criteria for design evaluation [16].

Donald Norman's [1993] points out through the situated cognition, that the human mind is limited in capacity even though the brain is a powerful information-processor, but there are limits to its capacity to remember, calculate, navigate, plan, and learn. According to his finding, human can overcome these limits, creating and using artifacts that scaffold them in performing cognitive tasks. According to Norman [1991], "personal view" and "system view" of cognitive artifacts are not same. Cognitive artifacts enhance cognition by allowing human to accomplish cognitive tasks faster and with less errors by transforming cognitive tasks. The research in this paper assumes this transformation as the base of integration of systems and design thinking. The rest of the paper is organized as follows. Section 2 briefly explains how the system could understand the abilitydemand gap in terms of the Gap Model [11]. Systems thinking and design thinking approaches that are used successfully in much research are explained. In addition, some related literatures, popular assistive apps and usability measures are explained. In section 3, an extension of the Gap Model for flexible disability assessment is articulated by adopting the system dynamics perspective in assistive thinking. In section 4, an approach to bridge the ability-demand gap through associative thinking is shown. Following this, Section 5 summarizes simulation results with cognitive scores from systems thinking and design thinking perspectives. Finally, section 6 concludes the paper with a few remarks on lessons learned and future works.

\section{Background}

According to World Health Organization (WHO) ${ }^{3}, 285$ million people are estimated to be visually impaired worldwide; 39 million are blind and 246 have low vision; About $90 \%$ of the world's visually impaired live in low-income settings; $82 \%$ of people living with blindness are aged 50 and above [3]. Globally, uncorrected refractive errors are the main cause of moderate and severe visual impairment; cataracts remain the leading cause of blindness in middle- and low-income countries. The number of visually impaired people from infectious diseases has reduced in the last 20 years according to global estimates work. $80 \%$ of all visual impairment can be prevented or cured. The assistive thinking approach starts with understanding the ability-demand gap of the user and investigates the level of support a user might need. This section reviews one example of a Gap Model for the

${ }^{3}$ Google Play Statistics: https://play.google.com 
sake of clarity and its application in the identification of the gap. It is also reviewing important concepts from systems thinking and design thinking that are critical components of assistive thinking. In addition, some popular mobile applications that can adopt this proposed framework in developing assistive solutions are also reviewed.

\section{Disability and Technology Interaction Gap}

Disability is defined as a condition or function judged to be significantly impaired relative to the usual standard of an individual or group [17]. The term, disability, is used to refer to individual functioning, including physical impairment, sensory impairment, cognitive impairment, intellectual impairment or mental illness, and various types of chronic disease. From a multidimensional standpoint of the person involved, there may be effects on organs or body parts as well as a person's participation in areas of life. Disabilities can be categorized as physical or sensory, cognitive, and developmental. Psychosocial or mental disorders and various types of chronic diseases may also qualify as cognitive and neural-disabilities. In universal design perspective, the gap created between a user's ability and the demands of the environment is also termed disability [11]. Figure 1 shows the Gap Model with elaboration of the gaps between user's different abilities and corresponding demand on the environment.

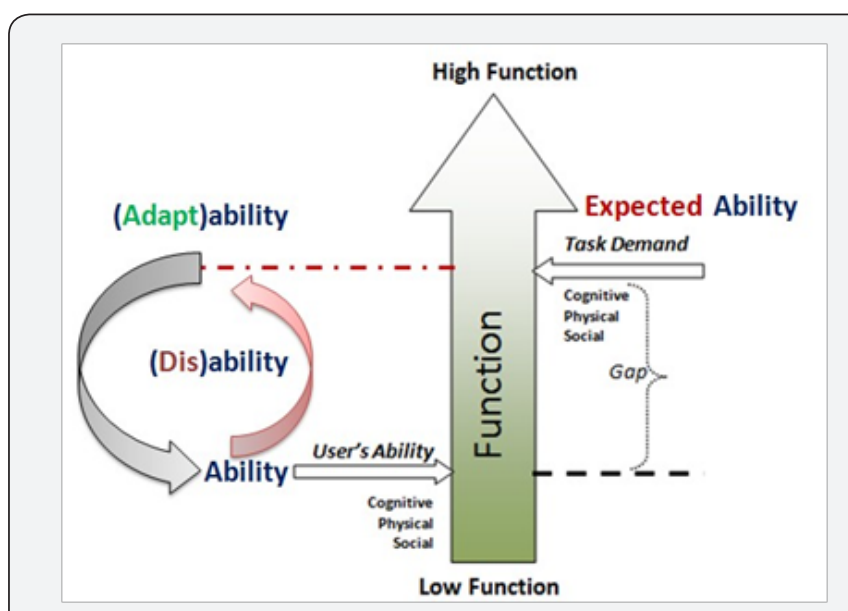

Figure 1: Schematic of the Gap Model [Aslaksen 1997] and its extension to Adaptability cycle.

\section{Current Practices in AT Applications}

Android applications have a mediocre performance in communications, navigation and day-to-day activities for people who have a disability $[3,18]$. Useful application can be considered as assistive apps as these work as a faithful friend to an individual with a disability. As of now, various assistive apps have been explored in the Android apps community ${ }^{4}$. Some instances of useful assistive apps are provided in this section to give a utilitarian perspective. For example, R-MAP [19-22] is a fully integrated, stand-alone system with easy-to-use interface to reconfigure an Android mobile phone. Trinetra was developed in CMU (Lanigan, 2006) as a barcode-based, cost-effective AT solution to provide blind people a greater degree of independence in their daily activities. Blind Shopping [19] a barcode and QR Reader [20] are the latest Android applications. The app is simple to use and all it requires from the user is to take a picture and let the app read the UPC and QR codes. To real time ansering visual question VizWiz system is developed (Bigham, 2010). Talk Back [21] is part of Google's Android Accessibility Service, designed to help blind and visually impaired users in using their mobile devices. Talk Back helps blind people hear what they are trying to do with their mobile phone by telling them the item that they have just selected or picked. Walky-talky [22] is one of the many navigation apps by the Eyes-Free Project that helps blind people get along with their daily lives, also supported the application developed Eye-Free navigation and browsing [23]. Despite the availability of these assistive applications, there is a need of generalized framework of analysis and modeling assistive applications. The general framework proposed in this paper can be regarded as an integration of both systems and design thinking approaches through users' cognitive load level [24]. We study how to continuously monitor users' cognitive load levels in accessing AT applications, we apply machine learning algorithms the have adaptive AT system, where user interfaces are assumed to admit users' cognitive gaps in interaction based on the cognitive load level. Hence, this research introduces an integrated general framework for representing this class of adaptive transductions by means of systems and design thinking.

\section{AT Systems Models}

The novel research on modeling AT solutions advances technologies with innovations and models. Social cognitive model [25] - suggest that human behavior is preceded by his intentions that are influenced by his attitude toward the behavior and the immediate social context. According to Carter [26], human personal attitude is driven by his perceived sentiment with positive/negative consequences of his behavior and modulated by his personal motivation. The influence of the social context effects to his surroundings - spouse, co-workers, and practitioners. The consideration model is proposed by Chamber [27]. The simple flowchart enabled consideration process model illustrates key questions and decisions that must be made when considering AT solutions. There are four key assessment factors in this model:

a) Currently effective instructions;

b) The knowledge base;

c) Interactions between

d) And

e) And

f) Updates to the user.

Chau and his collaborators proposed the technology and media (TAM) Model [28]. User's acceptance of computer technology can be predicted and explained through the model. According

${ }^{4}$ Official Android Design guidelines: http://developer.android.com/Design/index.html 
to Chau et al., the model can account the user perception on the usefulness of technology and attitude towards the use of the technology from the intensity of an individual's attention to use a technology. The AT CoPlanner Model [29] provides the basis to facilitate communication, collaboration, and co-planning of AT in the schools within a timely efficient manner. Four circular stages in CoPlanner model are:

a. Orientation-need assessment of AT service;

b. Assessment and Planning-gathering information about instruction and contex;

c. Implementation and Evaluation-mapping instruction in effective action; and

d. Reporting-determining whether a user should receive support or not.

A framework for conceptual modeling of AT device outcomes is proposed by [30].

The first event, the procurement of device-type, includes three considerations:

a. The need for an AT device;

b. Type of the AT device; and

c. The services involved with the device.

Around the same time, the device discontinuance model [31] proposed-aiming to classify the reasons for device discontinuance into different factors reflecting positive and negative discontinuance which is measured through two different scales: positive-negative scale and a factor of discontinuance scale. The factors of discontinuance are a complex interrelation of variables. Human Activity Assistive Technology (HAAT) Model is a framework that describes the major elements of an AT system (Cook \& Hussey 2002). In HAAT model, the human/technology interface allows communication between user and technology devices through:

a. The processor link and interpret information from the interface;

b. The environmental interface adjusts the output of device in response to the input from the environment; and

c. The activity outputs facilitate actions for the task or activity.

Hence, the four important parts of HAAT model are:

a. Activity

b. Context

c. Human

d. Assistive technologies

Details of summarized in Table 1 . The proposed assistive thinking is a cognitively inspired modeling technique that cogitates the gap between person's cognitive ability and AT system's cognitive demand-from the outcome of task difficulty vs. user's cognitive load. The modeling technique blends systems and design thinking in AT design and implementation perspectives and promises to improve communication gap between human and AT devices in different contexts (home, society, culture or physical). The model considers all activities of daily living, work and productive activities as the actuating component. Similarly, with sensory input and central processing concern in the environmental context. Hence, the thinking strategy aims to improve actuation and adaptive component of the HAAT model by cognitively facilitating actions for the task or the activity.

Table 1: Human Activity Assistive Technology (HAAT) Model [Cook \& Hussey 2002].

\begin{tabular}{|c|c|c|c|}
\hline Activity & Context & Human & $\begin{array}{c}\text { Assistive } \\
\text { Technologies }\end{array}$ \\
\hline $\begin{array}{c}\text { a) Activities } \\
\text { of daily living } \\
\text { (ADL), }\end{array}$ & $\begin{array}{c}\text { a) The setting (e.g., } \\
\text { at home, at work, in } \\
\text { the community), }\end{array}$ & $\begin{array}{c}\text { a) Sensory } \\
\text { input, }\end{array}$ & $\begin{array}{c}\text { a) The human/ } \\
\text { technology } \\
\text { interface, }\end{array}$ \\
\hline $\begin{array}{c}\text { b) Work and } \\
\text { productive } \\
\text { activities } \\
\text { (WPA), and }\end{array}$ & $\begin{array}{c}\text { b Social context } \\
\text { (with peers, with } \\
\text { strangers), }\end{array}$ & $\begin{array}{c}\text { b) Central } \\
\text { processin } \\
\text { g, and }\end{array}$ & $\begin{array}{c}\text { b) The } \\
\text { processor, }\end{array}$ \\
\hline $\begin{array}{c}\text { c)Play and } \\
\text { leisure (PL). }\end{array}$ & $\begin{array}{c}\text { C) Cultural context, } \\
\text { and }\end{array}$ & $\begin{array}{c}\text { c) } \\
\text { Effectors } \\
\text { (motor) }\end{array}$ & $\begin{array}{c}\text { c) The } \\
\text { environmental } \\
\text { interface, and }\end{array}$ \\
\hline & $\begin{array}{c}\text { d) Physical context } \\
\text { (light, sound, heat). }\end{array}$ & $\begin{array}{c}\text { d) The activity } \\
\text { output }\end{array}$ \\
\hline
\end{tabular}

\section{From user-Centered to Adaptation-Centered Systems}

Intelligent interaction system adaption requires to look at how well the system design is validated in the target community and to what extent the system supports universal accessibility. Recent study proposes an adaptation-centered systems design [32] in this purpose [33]. Also examined contemporary space systems, engineering procedures, and developed a systems engineering (SE) analysis tool for next-generation small satellites. The smart phone is being considered as a system integration tool in many cases, including highly adaptive technology solutions (e.g., AT solutions, smart home) [34]. There are eight tenets of systems thinking: interdependence, holistic, multi-finality, equi-finality, differentiation, regulation, abstraction, and leverage points to technology-based social ventures. These tenets are proposed in a concurrent design space like the E-sport canvas [35]. The relevance of eight tenets of systems thinking to the E-Spot canvas was proposed in [36], which is a design space and business strategy exploration tool that facilitates group thinking amongst stakeholders [37]. Argued that traditional systems analysis and design approaches are insufficient to model health care systems, given their complexity, the presence of uncertainty, and the scale. To overcome these difficulties, a system-of- systems approach is adopted (Ge, 2014). Systems-of-system methodology provides more flexibility and adaptability for the automated construction of executable models directly from the architectural data, rather than from static models (Ge, 2014). An advanced driver- 
assistance system that uses a low-cost embedded digital signal processor is proposed [38], with the overall system installed in a commercial vehicle [39]. Examine the problem of remote authentication in online learning environments and explore the challenges and options of using biometric technology to defend against user impersonation attacks by certifying the presence of the user in front of the computer at all times [40]. Proposed a Gaze-sensitive Adaptive Response Technology (VIGART), which is capable of delivering individualized feedback based on a child's dynamic gaze patterns during VR-based interaction. They performed a systematic usability analysis to evaluate the potential of this novel technology. As of now, various types of AT systems have been proposed. For example, Lip Control System (LCS) [41] is an innovative human-computer interface specially designed for people with tetraplegia. The authors evaluated the lower lip potential to control an input device, according to Fitts' law (ISO/ TS 9241-411:2012 standard). These recent research enterprises, encourage a new thinking approach involving the integration of both systems and design thinking in order to support the holistic approach of mobile phone based AT solutions.

\section{Systems Thinking Concepts}

Systems thinking considers cause and effect with an explanation of the necessity to deal with the complexity of the user's task and his interaction that can be explained with causal loops. Systems thinking optimizes performance of the overall system by describing the system, finding operation patterns (trends/dynamics), identifying variables, setting system boundaries, making the system visible and determining leverage points [7]. A popular systems thinking tool is the Causal Loop Diagrams (CLDs), that shows cause and effects and their links with system variables. CLDs help in eliciting and capturing users' mental models and describing the causes behind the activity dynamics. An example of a CLD can be, the human learning and cognitive processing system, is elaborated with CLD in Figure 2. In this example the variables are: cognition rate, learning, rate of forgetting, fractional cognition rate, and the average forgetting time. They are connected by 'causal links' (arrows) with assigned polarities $(+,-)$. Polarities indicate how the dependent variable are affected with the independent variables and loop identifiers, indicating the direction of circulation and types (balancing, B, or reinforcing, R).

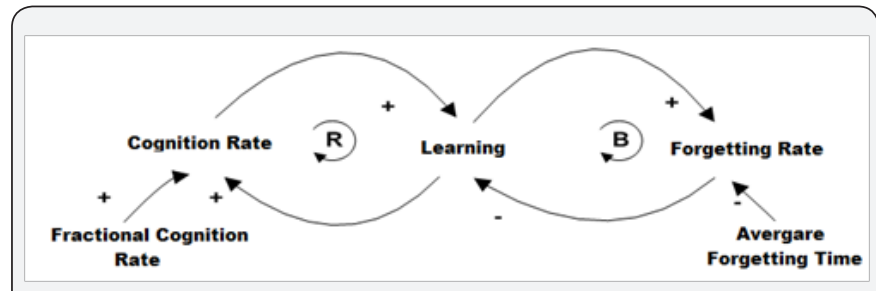

Figure 2: Causal Loop Diagram (CLD) of human memory and forgetting process.

System dynamics are an advanced systems thinking approach [1], constructs the same CLDs of systems with feedback and further utilizes simulation to study the behavior of systems and the impact of alternative policies. In system dynamics, the mental model represents the framing of the problem by using a network of cause and effect known as causal belief networks and describes how the system operates. Systems dynamics model helps the designer to see how the defined system structure behaves in time. A system dynamics approach of the human cognitive process is shown in Figure 3. Qualitative System Dynamics (QSD) [42] addresses the problem of accessibility by using human control of automation. System dynamics approach is used in understanding technology adoption in agribusiness management [43], in medical diagnostics to the early stage planning of a new stroke unit [36]. SD models are also useful managing interaction errors by simulating the impact of errors due to human behavior [44]. SD models can point different influences they adopt from these networks.

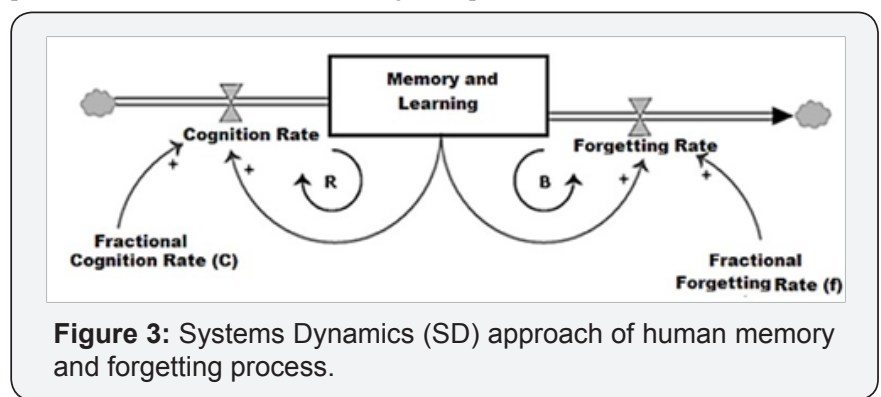

\section{Design Thinking Applications}

Design thinking is an optimal usability approach for UserCentered Design (UCD). It enables the deeper understanding of the user's need and mapping user interactions with the environment $[5,2]$. Instead of looking for an immediate solution to the problem, design thinking explores many possibilities. By this way, it creates an optimal solution for the stockholder. Like systems thinking, a design thinking approach strives to find user needs, looks for trends, identifies optimality criteria, sets design challenges, generates the solutions, and tests to realize the effectiveness. The design process deals with issues related to human behavior and psychology. For example, one design process could be concerned with how people respond to situations or react to a perceived problem. Design thinking approaches focus on how the user interaction with the system, gaps in user knowledge and practice, and ways to bridge the gap between what users know and what they need to know. User Sensitive Inclusive Design (USID) aims to design effective applications and accessible interfaces for elderly people, addressing the dynamic diversity [2]. Cognitive load analysis is indexed to measure the usability of the user interface as an integral part of effective interface design in several studies $[8,12]$. According to C. D. Wickens, a system can be modeled with the consideration of human mental resources and mental workload [45-47].

Real world systems are complex and heterogeneous. Recent progress in modeling dynamical processes helps to integrate realworld systems. Human dynamical influences in user interface interaction [47], socio-technical systems [48] and neural prosthetic system design [49] are some examples. More literature on Neurorehabilitation $[49,50]$ evident a demand of cognitively 
inspired new thinking process concerned to the modeling of AT solution. An example of UCD with individuals using AT is depicted in Figure 4. As an example - Smart Powerchair [51] is a System of Systems (SoS), in which pervasive technology is integrated into a standard powered wheelchair. But this model is not cognitively inspired. The proposed system focuses on the need of disabilities to understand a changing context or changing user abilities and to apply simulation and modelling for system dynamics understanding. Hence, this is the main contribution rather than System thinking, SoS and SE in general.

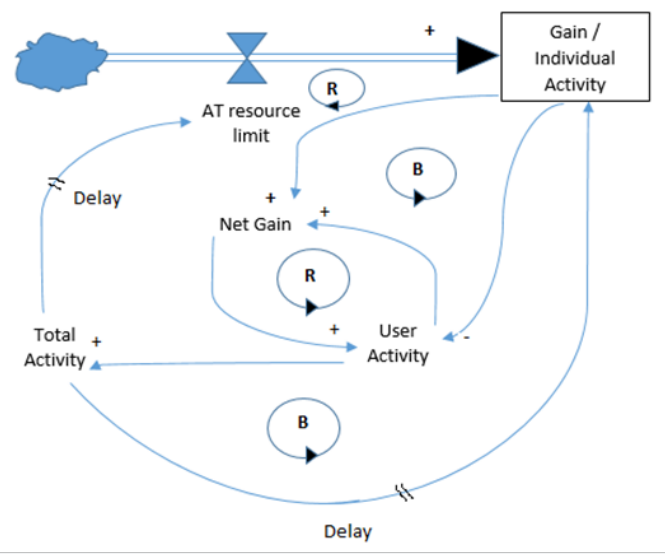

Figure 4: An example UCD for Net cognitive gain assessment from user activity and AT use.

\section{Assistive Thinking Approach}

Assistive thinking is intended to understand the user's abilitydemand gap in developing technology solutions and devise a plan for disability management. Assistive thinking compares user cognitive capabilities captured through both system and interface interactions and regulates user's decisions based on recommendations. Subsequent subsections discuss these issues for the sake of clarity. Due to poor system design and/or interface design a user might need more cognitive effort in performing AT operations. In both cases (system or interface/usability), user's extra cognitive effort experience the cognitive (over) load to the user. In assistive thinking, system access related cognitive effortcognitive load assessed through systems thinking (CLST) and user interface associated cognitive effort-cognitive load assessed through design thinking (CLDT) is categorized as HIGH - if it exceeds some threshold (inflection point [52], LOW - if it is equal or less than the threshold) and compared for decision support. This is illustrated through the left side of Figure 5.

\section{Systems Thinking with Assistive Component}

While the official android guidelines are provided ${ }^{5}$, special consideration is neither given in disability management nor in assistive application design and development [3]. Guidelines of effective AT, design [53-55], help to overcome this barrier with the new approach of cognitive gap reduction with assistive systems thinking approach. In systems thinking and dynamics system characteristic curve falls under three fundamental behavior models:

a. Exponential growth behavior - arises from positive or self-reinforcing feedback;

b. Goal seeking behavior - arises from negative or selfcontrolling feedback; and

c. Oscillation behavior - arises due to the positive or negative feedback, with significant time delays $[1,3,56]$.

The system thinking with assistive components that is called "assistive systems thinking [3]" (Figure 5) aims to optimize performance of the overall system, following the same concepts of systems thinking. It describes the critical components of the system, finds operational trends/dynamics, identifies variables, sets system boundaries, makes the system visible, and determines leverage points. In modeling operational trends of the system, user's cognitive capability and difficulties must be addressed. In addition to what is entailed in a system thinking approach, it generates valuable decision support by means of feedback to the user for technology adaptation purpose. The causal loop diagram (CLD) provides information about whether one variable causes the other with positive or negative effect. CLD does not provide the details of how this is caused and affected. The stock and flow diagram resolves the problem with three central entities:

a) Stocks - are the sources of delays, refer to accumulations, aggregations, summations over time;

b) Flows-represents a change of stocks with inflows and outflow direction; and

c) Feedback-proves the circular flow to show recurrent loops. Stocks also provide memory and give the inertia by accumulating past inflows.

In addition, it causes variations in oscillations over time by accumulating flows, decouple the inflows and outflows of a system [Sterman 2000].The development of stock and flow structure constructs a CLD with auxiliary and primary variables ${ }^{6}$.

\section{Design Thinking with Assistive Components}

The design thinking with assistive component that is called "Assistive design thinking" (Figure 5), expected to help the designer to develop an innovative user interface [3]. The main goal is to provide the best services and meaningful feedback at the right time. The assistive design thinking approach encompasses design thinking strategies based on high level usability analysis and engineering. It includes user interaction behaviors, gaps in user knowledge and practice, and ways to bridge the gap between what users know and what they need to know. Assistive design thinking analyzes needs, looks for trends, identifies optimality, sets design challenges, generates the solutions, and tests to realize

${ }^{5}$ World Health Organization (WHO): http://www.who.int/mediacentre/factsheets/fs282/en/

${ }^{6}$ Scan life- Mobile Barcode Trend Report: Holiday Shopping Edition- http://www.scanlife.com/en/ 
the effectiveness. In short, assistive design thinking is important for determining user need and the grasp usability of user interfaces, based on his/her cognitive capability and limitations that are reflected through the user's experienced cognitive load. Figure 6 (the right side) illustrates design thinking with cognitive load. As an important part of a usability study, understanding the areas of high cognitive load helps the designer to consider what factors are causing the high load in order to potentially redesign the interface to reduce the load. Thus, cognitive load can be measured through both channels and become a key metric for assistive thinking. Different combinations of assistive thinking scores recommend us both systematic and design aspects to be considered. For instance, high cognitive load from both channels, recommends any acceptable design of systems and interfaces, but all other combinations may be acceptable for AT users. During a needs assessment, a disability model can be helpful and be an integral part of the assistive thinking approach. For a systems thinking approach, frequently stock and flow diagrams help in understanding several aspects of the system. Nielsen's usability engineering approach can be applied in a subjective cognitive capability assessment as a part of assistive design thinking [16]. These are explained later on in Table 2.

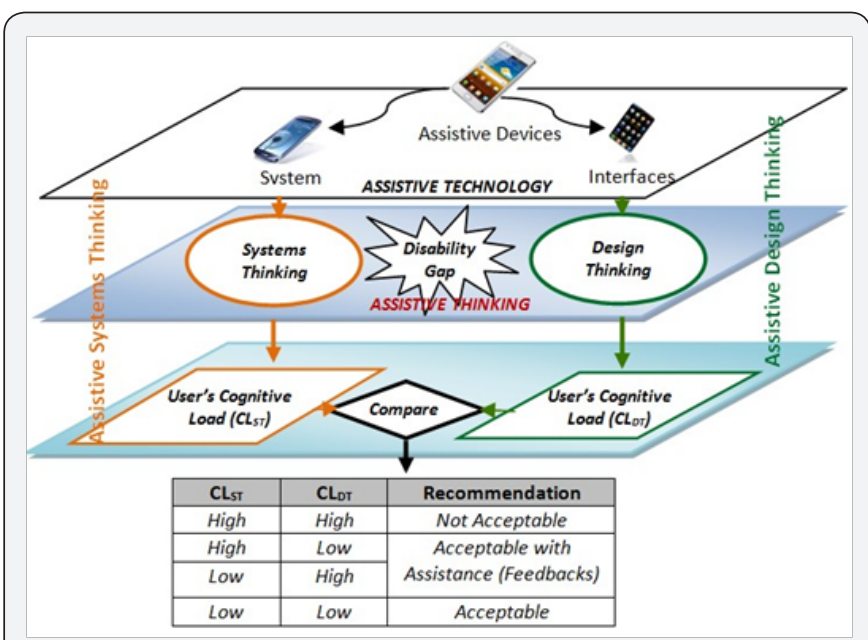

Figure 5: The Assistive Thinking Approach with systems and design thinking linked with cognitive load (CLs). High and Low are relative measures of cognitive load, depending on the specific load index.

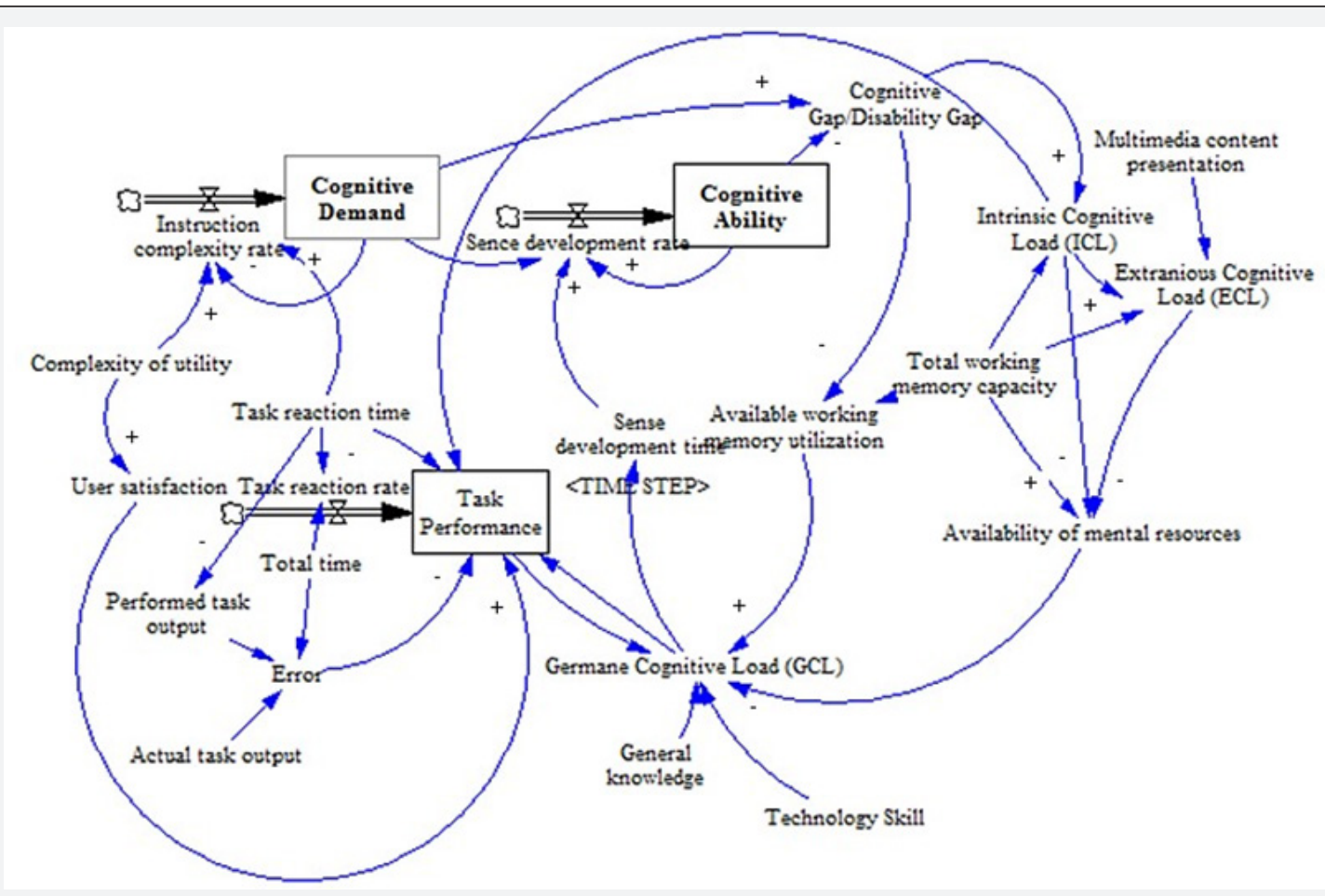

Figure 6: The assistive thinking model of disability; this model considers the cognitive demand and ability in cognitive task interaction [Hossain \& Yeasin 2013].

Table 2: NASA-TLX Used in Subjective Cognitive Load Computation.

\begin{tabular}{|c|c|}
\hline Scale & Description \\
\hline Mental Demand & $\begin{array}{r}\text { How much mental and perceptual activity was required (e.g. thinking, deciding, calculation, remembering, looking, searching, } \\
\text { etc.)? Was the task easy or hard, simple or complex, extracting or forgiving? }\end{array}$ \\
\hline $\begin{array}{c}\text { Physical Demand } \\
\text { How much physical activity was required (e.g., pressuring during tapping interface, tapping in different locations, double- } \\
\text { tapping, position the camera, positioning your hand, positioning the item etc.)? Was the task easy or hard, slow or fast, slack } \\
\text { or strenuous and restful or laborious? }\end{array}$ \\
\hline Temporal Demand & $\begin{array}{r}\text { How much time presser did you feel due to the rate or pace at which the task or task element occurred? Was the pace slow } \\
\text { and leisurely or rapid and frantic? }\end{array}$ \\
\hline
\end{tabular}




\begin{tabular}{|c|c|}
\hline Performance & $\begin{array}{c}\text { How successful do you think you were in accomplishing the goals of the task set by experimenter? How satisfied were you } \\
\text { with your performance in accomplishing these goals? }\end{array}$ \\
\hline Effort & How hard did you have to work (mentally and physically) to accomplish your level of performance? \\
\hline Frustration level & How insecure, discouraged, irritated, stressed, and annoyed versus secure, gratified, content, relaxed, and complacent did you \\
feel during the task?
\end{tabular}

\section{Assistive Thinking and Ability-Demand Gap}

By definition of the ability-demand gap, it can be formalized as the difference between cognitive ability of the user and the demand introduced by the system in performing a task [3]. AT system demand is a function of physical/sensory, psychological, and social demands in terms of task or event's complexities. Similarly, the individual's ability can also be defined by a function of his/her physical, psychological, and social abilities connected to his task or event's performance. Hence, the ability- demand gap can be calculated using the following formulas:

$$
\text { Demand }=\max \sum_{P s, P h d, S d} \text { Task complexity (1) }
$$

Where, $P d$ is the physical demand, $P h d$ is the psychological demand, and $S d$ is the social demand. Similarly, human ability can be defined using the formula:

$$
\text { Ability }=\max \sum_{P s, P h d, S d} \text { Task performance (2) }
$$

Where, $P d$ is the physical demand, Phd is the psychological demand, and $S d$ is the social demand. With demand and ability function, one can equate the ability-demand gap as a function of disability.

$$
\text { Disability }=F(\text { Demand }- \text { Ability })+K
$$

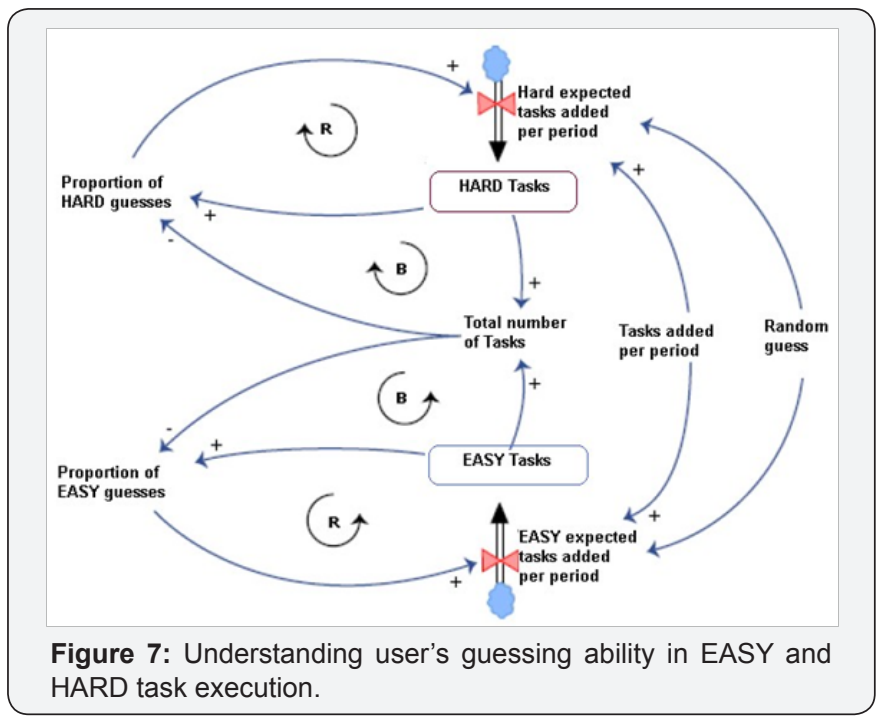

Where $\mathrm{K}$ can be considered as, person, specific unknown factors. Figure 6 illustrates the ability and the demand confluence with systems dynamics modeling with stock and flow diagram. In this model, intrinsic cognitive load (ICL), extraneous cognitive load (ECL) and germane cognitive load (GCL) are formulated and modeled with system dynamics modeling concept [7], which is proposed to apply in AT design as a part of assistive thinking. In the sketch of the model (Figure 6) contains three major stocks: cognitive demand (CD), cognitive ability (CA) and task performance (TP). Inflows and outflows from these stocks represent the cognitive loads and cognitive ability-demand gaps. For instance, the cognitive gap occurs from the differences between cognitive ability and demand, which is characterized with the available working memory resources. Accordingly, this gap results in an increase in ICL and ECL and a decrease in GCL. Given the task with difficulty level (EASY to HARD), user's ability to perform the given task requires, his proportion of guessing, the task complexity, time presser and environmental variables. Cognitive task performance is decomposed with task types performance and illustrated in Figure 7, the situation with both balanced and recurrent loops.

\section{Assistive Thinking with Systems and Design Thinking}

In mental modeling, one interesting research question arises, how a proposed system can predict the user's mental state, given the pattern of user activities? As an idealized mental (cognitive) model framework, Pólya urn model [10], which is named after George Pólya can be adopted. Polya model is a type of statistical model used, unifying many treatments and have many real life applications in random sampling [57,58]. Traditionally this type of modeling problem is solved with the sampling without replacement technique, where it is assumed that, every time a particular value is observed, it is less likely to be observed again [10]. Whereas, in a Pólya urn model, an observed value is more likely to be observed again, as like human information adaptation, learning from the environment. Hence, it is adopted in assistive thinking paradigm. It is noticeable that in a Pólya urn model that, successive acts of measurement over time have less and less effect on future measurements, unlike in sampling without replacement - after a certain number of measurements of a particular value, the value never been seen again $[58,59]$.

Finally, Figure 8 shows the placement of assistive thinking with steps of systems and design thinking. In an assistive thinking process, the system or design thinking is proposed to be aligned with the mental model to have the adaptive assistive component in it. The analogy of 'iceberg model' in assistive thinking (Figure 8) is adopted from $[9,14]$, to illustrate the conceptual modeling with four levels of thinking as a framework for systemic interventions. There are two major parts of the model: (a) visible and (b) invisible. The visible part contains only one level "events or symptoms" - those issues that are easily identifiable, in other word the level above the waterline. A user takes most of his decisions and interventions, which is represented at this level. What causes these events or symptoms are most of the invisible-under the water line. Within the rest three levels, analyzing the pattern of behavior works as an important issue in designing intelligent and adaptive technology system. 


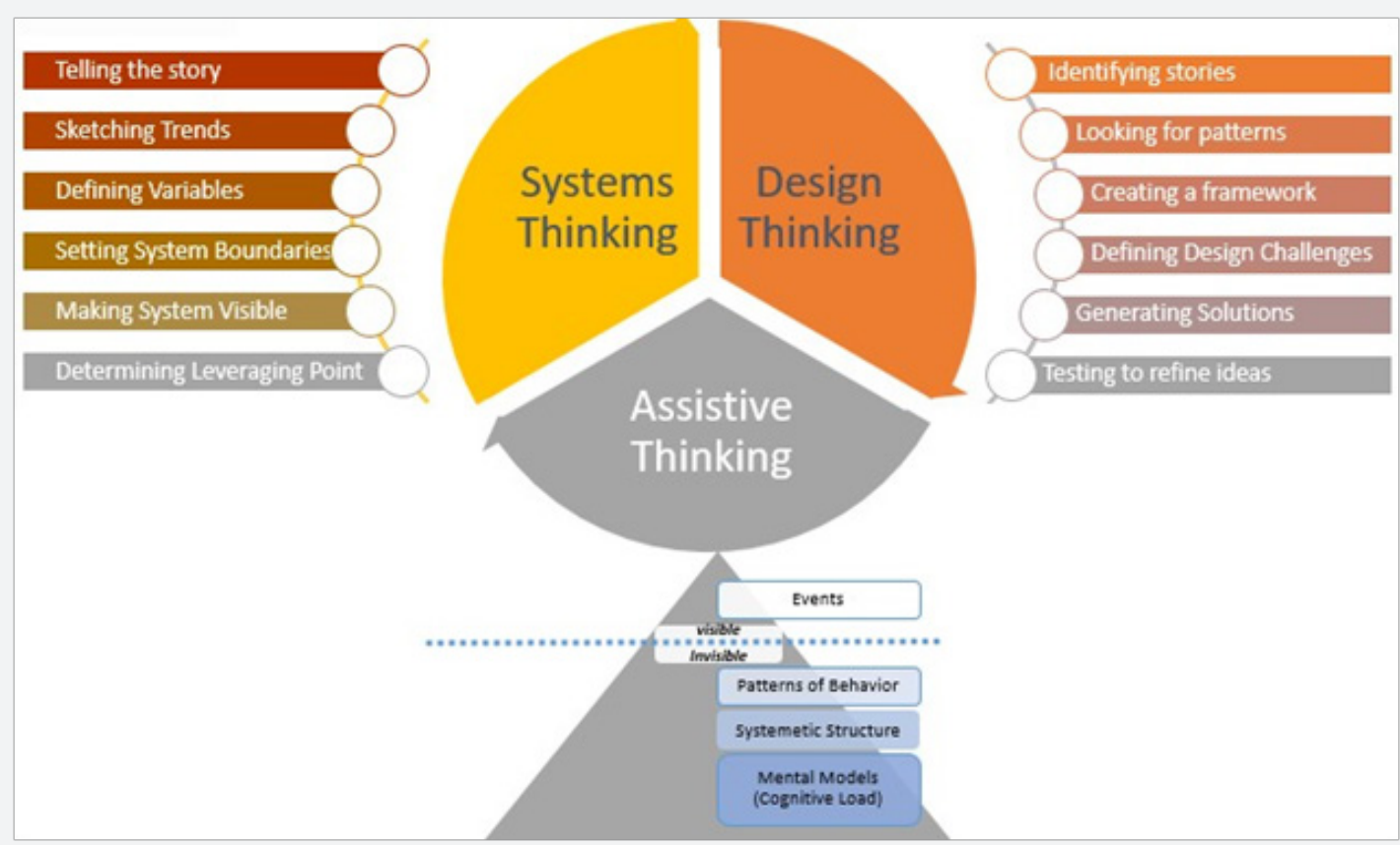

Figure 8: Conceptualization of Assistive Thinking (with 'iceberg' mental model adopted from [Mania \& Maharaja 2004; Mania \& Cavana 2007] and Polya urn model [Hoppe 1984]) connecting the systems thinking and design thinking. Sketching trends, looking for patterns and analyzing patterns of behavior joints the circular connection.

This is as because, treating the symptoms appear to be the easiest way out, even though the recent events are not always reliable. However, at the fourth level of the thinking, that hardly ever comes to the surface is considered as the 'mental models of individuals', influences why things work the way the users' do. The mental effort (in other word 'cognitive load') in mental models reflects the beliefs, values and assumptions that a user personally holds in cognitive task execution (e.g., Mobile application interaction) and he underlie his reasons for doing things the way he does [9]. The second important question arises, how does the Pólya urn process work in the assistive thinking framework? To answer such questions, let us assume, the urn contains balls of two colors, white and black. A ball is drawn at random and then put back in the urn along with a new ball of the same color. This process goes through several iterations. The two types of balls in the Pólya urn process [10] may represent two opposing choices that can be made about a certain aspect or activity in our lives (e.g. a task that assumes EASY or HARD in solving the problem). In a complex assistive context, problem solving activities accomplished through cell phone interaction may depend on guessing more on easy or hard task and to align with positive or negative re- enforcement (reward). What is the ratio of guessing easy to hard tasks in the user's mind after $\mathrm{N}$ (a number) tries? What is the ratio as the number of tries goes to infinity? The answer depends upon the initial number of easy and hard assumptions of the given tasks.

\section{Ability-Demand Gap Data Collection}

Categorical data are collected through a pretest of the experimental design using Nielsen's five usability attributes [16] (Table. 3).
Table 3: Three cognitive load questionnaires with usability study [Nielsen 1992].

\begin{tabular}{|c|c|}
\hline Question category & Question \\
\hline $\begin{array}{l}\text { Memorability (Intrinsic } \\
\text { Load : IL ) }\end{array}$ & $\begin{array}{l}\text { How difficult was the experiment instruction } \\
\text { content for you? }\end{array}$ \\
\hline $\begin{array}{l}\text { Learnability } \\
\text { (Extraneous Load : EL) }\end{array}$ & $\begin{array}{l}\text { How difficult was to learn with the } \\
\text { instruction format? }\end{array}$ \\
\hline $\begin{array}{l}\text { Efficiency (Germane } \\
\text { Load : GL) }\end{array}$ & $\begin{array}{l}\text { How much did you concentrate during } \\
\text { experiment? }\end{array}$ \\
\hline Errors & $\begin{array}{l}\text { What do you think about the chances of } \\
\text { errors during the experiment? }\end{array}$ \\
\hline Satisfaction & $\begin{array}{l}\text { How pleasant are you to participate in this } \\
\text { experiment and to use the design? }\end{array}$ \\
\hline
\end{tabular}

In computing user's cognitive load, and considering three cognitive load evaluations: Intrinsic Cognitive Load (ICL), Extraneous Cognitive Load (ECL), and Germane Cognitive Load (GCL). In an ICL assessment, the question considered is, "How difficult was the experiment instruction content for you?" In terms of this usability metric, it corresponds to the 'memorability' score, explaining how easily a user can reestablish proficiency when he returns to his interaction. In an ECL assessment, the considered the question is, "How difficult was it for you to learn with the instruction format?" This maps the 'learnability' in terms of how easy is it for users to accomplish basic tasks the first time they encountered the design. The GCL is considered through the question "How much did you concentrate during experiment?" This relates to the user 'efficiency' score of usability, which explains how quickly he/she can perform the learned tasks. All five questionnaires, including ICL, ECL, and GCL, are summarized in Table 2. According to the theory of cognitive load assessment, 
each type of cognitive load is additive [4]. Thus, total cognitive load (CLT) is calculated as follows:

$$
\text { CLTotal }=C L I+C L E+(-C L G)(4)
$$

Where, CLTotal is the total cognitive load, $C L I$ is Intrinsic, CLE extraneous, and $C L G$ refers to germane cognitive load. In assistive android application (the R-MAP [15]) development, cognitive load assessment adopted the NASA Task mental workload Load Index [13] with six scales to assess the mental workload: mental demand, physical demand, temporal demand, performance, effort, and frustration [60]. Table 4 shows the description of NASA-TLX dimensions. NASA-TLX takes on the approach of using subjective quantitative data. Five step graded response scales are used to obtain ratings for these dimensions (Very low to very high). A score from 0 to 10 is obtained with a 2-point increment on each scale. The six individual scale ratings are combined using users' self-emphasized weighting procedure- the user is asked to weight on his scale value (Cao 2009). A cumulative workload score from 0 to 1 is obtained for each rated task by multiplying the weight of the individual dimension scale score, summing across scales, and dividing by individual average score the normalized score is computed. Recent work of mental workload can also be found in $[3,24]$. Once the cognitive load is assessed through some measures from systems thinking and design thinking, the iceberg mental model is constructed by a Ploya process in mental modeling stage. Table 4 shows an example of quantitative and qualitative data collection questionnaires [61-65].

Table 4: Example of Quantitative and Qualitative Data in R-MAP case study.

\begin{tabular}{|c|c|c|}
\hline \multirow{2}{*}{ Objective } & $\begin{array}{c}\text { Quantitative } \\
\text { "R-MAP has accuracy of } \\
\text { in bottled surface text } \\
\text { recognition." }\end{array}$ & $\begin{array}{c}\text { Qualitative } \\
\text { "Mr. John seems happy } \\
\text { while using the R-MAP" }\end{array}$ \\
\hline Subjective & $\begin{array}{c}\text { "On the scale of 1 to 10, John } \\
\text { considers 9 for R-MAP ease } \\
\text { of use." }\end{array}$ & $\begin{array}{c}\text { "John thinks R-MAP is } \\
\text { very useful for him" }\end{array}$ \\
\hline
\end{tabular}

\section{Results}

This paper presents the conceptual outline of an integrated approach to AT design and development. The empirical analysis clarifies the effects of cognitive behavior, usability, and technological acceptance of disabilities, along with an improved conceptualization of the impact of abstract variables on reengineering the AT solution. Methods of the research include analysis of qualitative (user observation) and quantitative data (subjective rating) from cognitive load and usability analysis. Tables 2 \& 4 shows data collection questioners. The application of assistive thinking approach in R-MAP interaction is also presented. The proposed design model, tuned with simulation data, and cognitive load were compared using subjective usability scores collected from 24 users (combination of people who are blind and blindfolded) of R-MAP interaction, which is adopted from the authors' earlier work $[3,24,52]$. Cognitive load simulation with the concept of systems thinking is followed from [12]. Only cognitive task demand and cognitive ability of a user has been considered, while computing the cognitive load. Physical demand and ability can also be modeled in a similar manner with computation of cognitive gap. While the cognitive load (i.e., A Gap in ability-demand) is simulated in a systems thinking process, it is also computed from usability questioners (alternatively can be considered from mental workload dataset). A case study was performed on the human cognitive behavior [66-69].

Three types of cognitive loads (intrinsic, extraneous and germane) were considered in the systems dynamic modeling with Vensim $\AA$ PLE (Sterman 1997). The simulated assistive thinking model is a cognitive load based design model, which accounts intrinsic, extraneous and germane cognitive loads in user's cognitive gap computation. The model is followed from a cognitive load dynamics model (Sawicka 2004). As an important consideration of the design thinking approach, all the three types of cognitive loads are computed from post-experimental (technology interaction) scores. The total cognitive load is computed by equation 4 . In the next step, computing the average cognitive load (equation 5), a comparative load graph of all users and different tasks are graphically illustrated (Figure 9). 24 users among them 20 blindfolded and 4 blind had participated in RMAP [15], the study.

$$
\text { CLcomp }=\text { CLtotal }+ \text { CLAvg }
$$

Where, CLcomp is the comparative cognitive load, CLTotal is the overall cognitive load (total load) and CLAvgl represents the average cognitive load. This work proposed an extension of the ability-demand gap that relates to (dis)ability. Given the task difficulty represents the user's ability in terms reaction time, the more time a user spends over a task, the more cognitive ability-demand gap he might have [3,52]. Intrinsic, extraneous and germane cognitive load is hypothetically plotted in terms of ability-demand gap and WM capacity. The simulation is performed with the following initial conditions: Total working memory (WM) capacity $=1$, Total_Cognitive_load=1, using equation number 4 . The output of the simulation is illustrated in Figure 9A. The green graph in Figure 9A corresponds to the extraneous load of the user, which, in this case, is a nonlinear curve. In contrast, the blue and red curves correspond to the germane and intrinsic loads, respectively. Both of them are approximately linear up to $20 \mathrm{Sec}$, indicating user's cognitive control on device, which is based on to the working memory capacity and cognitive gap. Again, Figure 9A \& 9B show the relationship of cognitive load, working memory capacity and their confluences. The simulation results of the intrinsic and germane load show similar trends, while extraneous load varies with a variety of instructional presentations (e.g., R-MAP camera operation may have two different ways). The confluence of the cognitive load (gap) from different channels (system and design) is then compared to provide a justification for a decision [70-72]. 


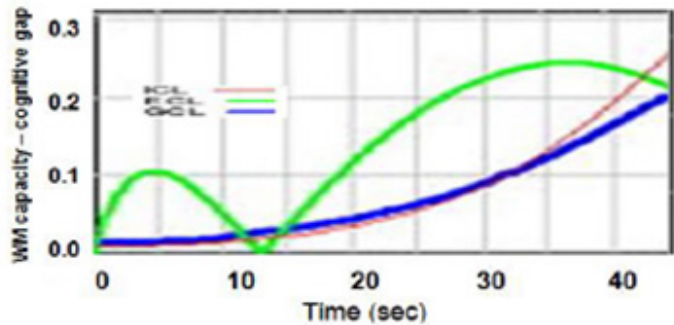

(A)

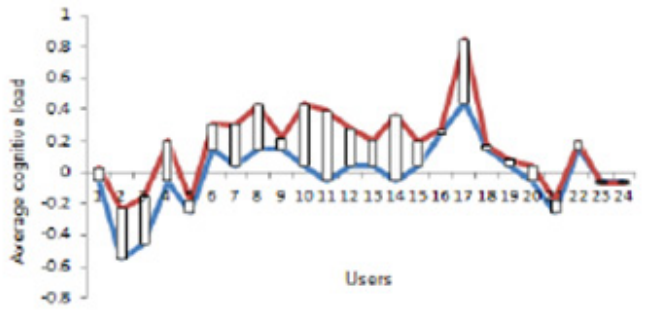

(C)

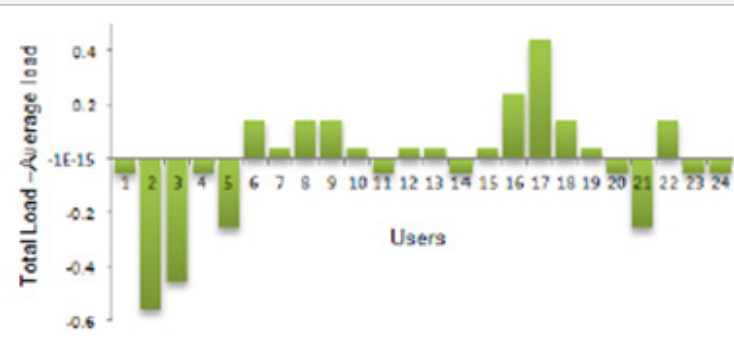

(B)

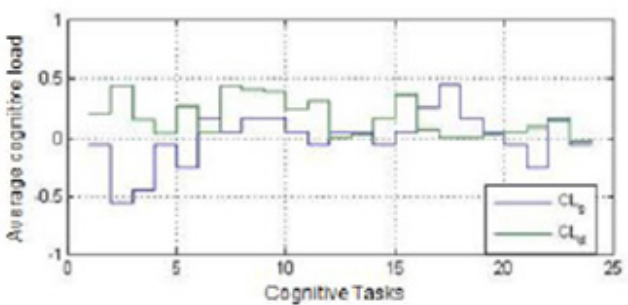

(D)

Figure 9: Cognitive load assessment and comparison. (A) Intrinsic (red), Extraneous (green) and Germane (blue) cognitive load (B) Cognitive load comparison from overload and average load (C) Cognitive gap in user centered design; The 'blue' line indicates CL from design perspective, the 'red' line is from systemic load assessment (simulated) and the boxes indicate differences between two cognitive assessments, (D) Comparison of average cognitive loads in the cognitive task based interaction (simulated); The 'blue' line indicates CL from design perspective, and the 'green' line is from systemic load assessment (simulated).

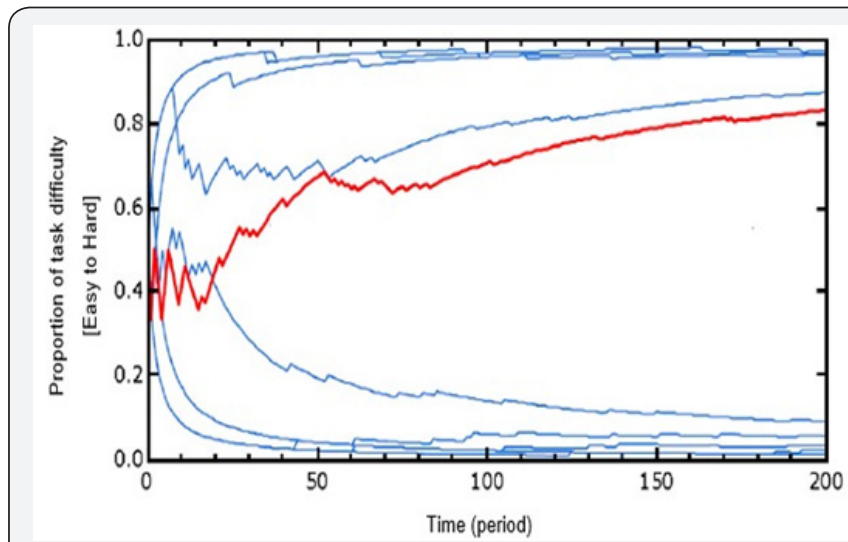

Figure 10: Understanding the users' intent of HARD tasks guesses with dominance.

The average cognitive load, which can be achieved from both cases, gives an important indication (trend) of the user-centered design (Figure 10) and cognitive ability design (Figure 9C), which are useful for further analyses, such as data mining and machine learning for user adoption. Boxes in Figure 9C, illustrates the average cognitive load in assistive systems thinking (blue) and assistive design thinking perspective (red), the difference indicates the cognitive gap, which is a useful measure in assistive thinking illustration. Figure9D represents a comparative assessment of users' cognitive ability for different task interactions. It is useful for cognitive ability-based design modeling. The bold, red line in Figure 10 shows that a run of HARD tasks changes an initial EASY task proportion to the HARD task dominance. According to Pólya, the process always converges to a fixed proportion of HARD tasks and the particular proportion depends on early history. All proportions of HARD tasks are equally likely in the long run. In general, the rules for determining flow are non-functions. Suppose the likelihood of choosing a HARD guess is determined by a nonlinear fraction. When the proportion of the HARD task rises above half of the likelihood of choosing a HARD task, then the chance of guessing a HARD task rises by more than $50 \%$. On the other hand, if the proportion is lower than one half, then the chance of choosing a HARD task is much lower than 50\%. This signifies that AT can be designed with the Polya's approach to benefit system adaptability [73-75].

\section{Conclusion}

Proposed research aims to design intelligent technology system to create value by fulfilling the needs of disabilities. Integrating people, technologies, organizations, and information the intelligent system will be capable to identify, learn, adapt, monitor and make decisions in fulfilling the needs. The system will utilize data sensed, received, processed and actuated in a timely manner, thus improving its response to future situations in adaptation. These capabilities will advance future AT systems towards collaborative systems with the result from incorporation of technologies for sensing, actuation, coordination, communication, and adaptation. The proposed assistive thinking framework integrates systems and design thinking with assistive components in terms of mental state modeling (cognitive effort) and decision support generation. The systems thinking with mental state modeling approaches have common core concepts applicable to all universal accessibility problems. The AT designer can benefit 
from early feedback about their system design improvement. The concept of systems thinking about AT, design and the design thinking for user interface design is combined with a new way of mental modeling which a paradigm shift in designing AT solutions. The proposed approach "assistive thinking" can also be named as "Design for disabilities" like "design for manufacturing", "design for kids" etc as examples. A new method for identifying user requirements for a particular user Group that may be integrated in a common front-end analysis and design process.

This paper has presented a model of how a future system can be designed with the facility to make sense of instantaneous ability- demand gap (disability), along with the cognitive load perspective. To support assistive thinking ideas with cognitive load, a framework of cognitive ability-based AT simulation and evaluation is proposed. How does the cognitive effort estimation can be a key leverage point in integrating systems and design thinking, also illustrated. Finally, using assistive thinking, an integrated (cognitive) thinking approach is proposed to better understand the disability gap/cognitive gap, and bridging the gap for successful communication. Assistive Thinking helps, in grasping AT trends, which are shown through the case study of an R-MAP user interaction with an Android platform. Negative results in Figure 9B-9D indicate some inconsistencies in average cognitive load score depending on the user's ability, and visually illustrate the requirement of associative thinking. Further effort will be put in mining patterns of user-centered design / cognitive ability-based design in technology based disability management. Real time user-centric cognitive load assessment techniques will be considered [54] towards a real time Assistive Thinking simulated with Neuro-cognitive, physiological signals (EEG, ECG, HRV etc.).

Further research is needed in order to test this model and explore how thinking processes impact other elements in the disability management system. More simulation and validation with real data and combining all aspects of gap modeling might be necessary. By improving the understanding of these dynamics, it will have great potential for improving navigation, personal adaptation, communication, and managing the gap in interpersonal social coordination. To help with the paradigm shift, following investigations are recommended:

a. To have full potentials of adaptive personalize technology systems, more innovative ideas out there that may accomplish even more than discussed above can be researched

b. More investigations needed to the transformation methods that can help with the transition while allowing communities to continue develop application using systems and design thinking concepts

c. Integration of various technology and supporting devices (e.g., google glass, fitbit, hearing-aids etc.) and implicit parallel paradigm with heterogeneous systems to work with multiple interconnection networks at the same time d. To investigate light weight version of systems thinking and design thinking and modeling technology solutions with both and their impact in assistive thinking with

e. To investigate the possibility of assistive thinking approach in tuning for HPC cloud based systems.

\section{Acknowledgments}

The authors would like to acknowledge Google Play, Disabled World, WHO and Official android design guidelines; thanks to J. D. Sterman for his instructional guideline (book) for systems thinking concepts and simulation tools. This work is partially funded by NSF grant number NSF-IIS-0746790.

\section{References}

1. Sterman JD (2000) Business dynamics: systems thinking and modeling for a complex world. Irwin McGraw-Hill, Boston, USA.

2. Brown T (2008) Design thinking. Harvard business review 86(6): 84 .

3. Hossain G, Yeasin M (2013) Assistive thinking: Assistive technology design in disability management. In Proc 1st Int Conf Technol, Helping People Special Needs (ICTHP) Riyadh, KSA, p: 18-20.

4. Checkland P(1981) Systems thinking, systems practice.

5. Rowe PG (1991) Design thinking. MIT press, USA.

6. Kelley T, Allender L (1995) Why choose? A process approach to usability testing. Advances in Human Factors/Ergonomics 20: 393398.

7. Pourdehnad J, Wilson D, Wexler E (2011) Systems \& design thinking: A conceptual framework for their integration. $55^{\text {th }}$ Annual Meeting of the ISSS-2011, Hull, UK 55(1): 1-16.

8. Dym CL, Agogino AM, Eris O, FreyDD, Leifer LJ (2005) Engineering design thinking, teaching, and learning. Journal of Engineering Education 94(1): 103-120.

9. Mania KE, Cavana RY (2007) Systems thinking, system dynamics: Managing change and complexity. Auckland, NZ, Prentice Hall, USA.

10. Hoppe FM (1984) Pólya-like urns and the Ewens' sampling formula. Journal of Mathematical Biology 20(1): 91-94.

11. Aslaksen F, Bergh, S Bringa OR, and Heggem EK (1997) Universal design: Planning and design for all. Cornell University ILR School, USA.

12. Sawicka A, Molkenthin R (2005) Cognitive load dynamics: How to increase effectiveness of SD-based learning environments. $23^{\text {rd }}$ Int Conference of the System Dynamics Society.

13. Hart SG, Staveland LE (1988) Development of NASA-TLX (Task Load Index): Results of empirical and theoretical research. Advances in psychology 52: 139-183.

14. Mania K, Maharaja V (2004) Links between systems thinking and complex decision-making. System Dynamics Review 20(1): 21-48.

15. Shaik AS, Hossain G, Yeasin M (2010) Design, development and performance evaluation of reconfigured mobile Android phone for people who are blind or visually impaired. In Proceedings of the $28^{\text {th }}$ ACM International Conference, Design of Communication, USA, pp. 159-166.

16. Nielsen J (1992) The usability engineering life cycle. Computer, USA 25(3):12-22.

17. Ryan C, Tewey B, Newman S, Turner T, Jaeger RJ (2004) Estimating research productivity and quality in assistive technology: a bibliometric analysis spanning four decades. Neural Systems and Rehabilitation Engineering, IEEE Transactions 12(4): 422-429. 
18. Fulcher R Nesladek C Palmer J Robertson C (2010) Android UI design patterns.

19. López de Ipiña D, Lorido T, López U (2011) Blindshopping: enabling accessible shopping for visually impaired people through mobile technologies. In Toward Useful Services for Elderly and People with Disabilities, Springer Berlin Heidelberg, Spain, pp. 266-270.

20. Skeele R (2013) QR Codes: Repurposing Technologies into Assistive Technologies. In Society for Information Technology and Teacher Education International Conference 1: 4060-4067.

21. Rodrigues A, Montague K, Nicolau H, Guerreiro T (2015) Getting smart phones to talkback: understanding the smart phone adoption process of blind users. In Proceedings of the $17^{\text {th }}$ international ACM SIGACCESS Conference, ACM, USA.

22. Angin P, Bhargava BK (2011) Real-time mobile-cloud computing for context- aware blind navigation. Int Journal of Next-Generation Computing 2(2): 405- 414.

23. Raman TV, Chen CL (2009) Eyes-Free User Interaction.

24. Hossain G, Shaik AS, Yeasin M (2011) Cognitive load and usability analysis of R-MAP for the people who are blind or visual impaired. $29^{\text {th }}$ SIGDOC, ACM, USA, pp. 137-144.

25. Holt EB, HC Brown (1931) Animal drive and the learning process, an essay toward radical empiricism. H Holt and Company, New York, USA.

26. Roelands M, Van Oost P, Depoorter A, Buysse A (2002) A socialcognitive model to predict the use of assistive devices for mobility and self-care in elderly people. The Gerontologist 42(1): 39-50.

27. Chambers A C (1997) Has Technology Been Considered? A Guide for IEP Teams. CASE/TAM Assistive Technology Policy and Practice Series. Council of Administrators of Special Education, Albuquerque, NM, USA.

28. Chau PYK, Hu PJH (2001) Information technology acceptance by individual professionals: A model comparison approach. Decision Sciences 32(4): 699-719.

29. Haines L, Sanche B (2000) Assessment models and software support for assistive technology teams. Assessment for Effective Intervention 25(4): 291-305.

30. Fuhrer MJ, Jutai J W, Scherer M J, De Ruyter F (2003) A framework for the conceptual modeling of assistive technology device outcomes. Disability and Rehabilitation 25(22): 1243-1251.

31. Lauer A (2004) Measuring positive and negative factors of device discontinuance. Unpublished Masters Thesis, University of Wisconsin, Milwaukee, USA.

32. Chilana PK, Ko AJ, Wobbrock J (2015) From User-Centered to AdoptionCentered Design: A Case Study of an HCI Research Innovation Becoming a Product.

33. Ekpo SC, George D (2013) A system engineering analysis of highly adaptive small satellites. Systems Journal, IEEE 7(4): 642-648.

34. Elhussein K, Babiker M (2013) Smart phones as system integration development tools:(Android SCL simulation environment as prototype). In Computing, Electrical and Electronics Engineering (ICCEEE), International Conference pp. 280-286.

35. Dzombak R, Mehta C, Mehta K, Bilen SG (2013) The praxis of systems thinking for concurrent design space and business strategy exploration. In Global Humanitarian Technology Conference (GHTC) pp. 438-446.

36. Elf M, Putilova M, von Koch L, Öhrn K (2007) Using system dynamics for collaborative design: a case study. BMC health services research $7(1): 123$.

37. Grigoroudis E, Phillis Y (2013) Modeling healthcare system-ofsystems: a mathematical programming approach. Systems Journal, IEEE 7(4): 571-580.
38. Chiang HH, Chen YL, Wu BF, LeeTT (2014) Embedded Driver-Assistance System Using Multiple Sensors for Safe Overtaking Maneuver. Systems Journal, IEEE 8(3): 681-698.

39. Moini A, Madni AM (2009) Leveraging biometrics for user authentication in online learning: a systems perspective. Systems Journal, IEEE 3(4): 469-476.

40. Lahiri U, Warren Z, Sarkar N (2011) Design of a gaze-sensitive virtual social interactive system for children with autism. Neural System and Rehabilitation Engg, IEEE Transactions 19(4): 443-452.

41. Archajo Jose M, de Deus Lopes R (2015) Human-Computer Interface Controlled by the Lip. Biomedical and Health Informatics, IEEE Journal 19(1): 302-308.

42. White AS (2011) Qualitative System Dynamics as a Tool in Accessible Design. Journal of Software Engineering and Applications 4(01): 6980 .

43. Fisher DK, Norvell J, Sonka S, Nelson MJ (2000) Understanding technology adoption through system dynamics modeling: implications for agribusiness management. The International Food and Agribusiness Management Review 3(3): 281-296.

44. Lee S, Peña Mora F, Park M (2005) Dynamic Error and Change Management in Concurrent Design and Construction. In Construction Research Congress, Broadening Perspectives, ASCE, USA, pp. 1-10.

45. Wickens CD (2002) Multiple resources and performance prediction. Theoretical Issues in Ergonomics Science 3(2): 159-177.

46. Wickens CD (2008) Multiple resources and mental workload. Human Factors. The Journal of the Human Factors and Ergonomics Society 50(3): 449-455.

47. Pan W, Cebrian M, Dong W, Kim T, Fowler J (2010) Modeling dynamical influence in human interaction patterns.

48. Vespignani A (2012) Modelling dynamical processes in complex sociotechnical systems. Nature Physics 8(1): 32-39.

49. Shenoy KV, Kaufman MT, Sahani M, Churchland MM (2011) A dynamical systems view of motor preparation: implications for neural prosthetic system design. Progress in brain research 192: 33-58.

50. Friederich A, Tanja B, Luc De W (2010) Methods for the selection of assistive technology in neurological rehabilitation practice. Scandinavian journal of occupational therapy 17(4): 308-318.

51. Whittington P, Dogan H (2015) Smart Powerchair: A pervasive system of systems. In System of Systems Engineering Conference (SoSE), IEEE pp. 244-249.

52. Hossain G, Yeasin M (2014) Cognitive Ability-Demand Gap Analysis with Latent Response Models. IEEE Transactions, ACCESS 2: 711-724.

53. Carmien S (2004) Design and Evaluation of Complex Assistive Technology Systems Using Ethnographic Methods.

54. Oviatt S, Coulston R, Lunsford R (2004) When do we interact multimodally?: cognitive load and multimodal communication patterns. In Proceedings of the $6^{\text {th }}$ international conference, Multimodal interfaces, ACM, USA, pp. 129-136.

55. Cooper R (1998) Harmonization of assistive technology standards. In Engineering in Medicine and Biology Society. In $20^{\text {th }}$ Annual International Conference of the IEEE 6: 3367-3369.

56. Agarwal RP, Bohner M, Cheung, Grace WS, Grace SR (2007) Oscillation criteria for first and second order forced difference equations with mixed nonlinearities. Mathematical and Computer Modelling 45(7): 965-973.

57. Banerjee A, Burlina P, Alajaji F (1999) Image segmentation and labeling using the Pólya urn model. Image Processing, IEEE Transactions 8(9): 1243-1253. 
58. Chen F (2004) An extended urn model with application to approximation. Transactions of the American Mathematical Society 356(9): 3505-3515

59. Chen M R, Kuba M (2013) On generalized Pólya urn models. Jrnl of App Prob 50(4): 1169-1186.

60. Hart SG (2006) NASA-task load index (NASA-TLX); 20 years later. In Proceedings of the human factors and ergonomics society annua meeting. Sage Publications 50(9): 904-908.

61. Alajaji F, Fuja T (1994) A communication channel modeled on contagion. Information Theory IEEE Transactions 40(6): 2035-2041.

62. Bernd T, Van Der Pij, De Witte LP (2009) Existing models and instruments for the selection of assistive technology in rehabilitation practice. Scandinavian Journal of Occ Therapy 16(3): 146-158.

63. Cao A, Chintamani KK, Pandya AK, Ellis RD (2009) NASA TLX: Software for assessing subjective mental workload. Behavior research methods 41(1): 113-117.

64. Carmien S (2005) End user programming and context responsiveness in handheld prompting systems for persons with cognitive disabilities and caregivers. In CHI'05, Human factors in computing systems, ACM USA, pp. 1252-1255.

65. Cook A, Arnold H S (2002) Assistive Technologies: Principles and Practices. Mosby Year Book Inc MO, USA.

66. Garrity Edward J (2012) Tragedy of the commons, business growth and the fundamental sustainability problem. Sustainability $4(10)$ : 2443-2471.

67. Ge B Hipel KW Yang K Chen Y (2014) A novel executable modeling approach for system-of-systems architecture. Systems Journal, IEEE 8(1): 4-13.

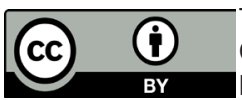

This work is licensed under Creative

Commons Attribution 4.0 License

DOI: 10.19080/JOJNHC.2018.06.555692
68. Gipson P, King C (2012) Health behavior theories and research: Implications for suicidal individuals' treatment linkage and adherence. Cognitive and behavioral practice 19(2): 209-217.

69. Haapalainen E, Kim S, Forlizzi JF, Dey AK (2010) Psycho-physiological measures for assessing cognitive load. In $12^{\text {th }}$ ACM international conference, Ubiquitous computing, USA, pp. 301-310.

70. Newell AF, Gregor P (2000) User sensitive inclusive design-in search of a new paradigm. In Proceedings on the 2000 conference on Universal Usability, USA, pp. 39-44

71. Norman D (1991) Cognitive artifacts. In; Carroll JM (Eds.), Designing interaction, Psychology at the human computer interface, Cambridge University Press, Cambridge, USA, p. 17-38.

72. Norman D (1993) Things that make us smart: Defending human attributes in the age of the machine. Basic Books, New York, USA.

73. Rauniar R, Rawski G, Yang J, Johnson B (2014) Technology acceptance model (TAM) and social media usage: an empirical study on Face book. Journl of Enterprise Info Management 27(1): 6-30.

74. Tatnall A, Anthony G (2005) Actor-Network Theory in Information Systems Research, pp. 42-46.

75. Wing JM (2006) Computational thinking. Communications of the ACM 49(3): 33-35

76. Zhu T (2009) Nonlinear Pòlya urn models and self-organizing processes (Doctoral dissertation, University of Pennsylvania, Philadelphia.

\section{Your next submission with Juniper Publishers will reach you the below assets}

- Quality Editorial service

- Swift Peer Review

- Reprints availability

- E-prints Service

- Manuscript Podcast for convenient understanding

- Global attainment for your research

- Manuscript accessibility in different formats

( Pdf, E-pub, Full Text, Audio)

- Unceasing customer service

Track the below URL for one-step submission https://juniperpublishers.com/online-submission.php 